\title{
Regularized direct and indirect symmetric variational BIE formulations for three-dimensional elasticity. ${ }^{1}$
}

\author{
Marc BonneT \\ Laboratoire de Mécanique des Solides (URA CNRS 317) \\ Ecole Polytechnique, 91128 Palaiseau Cedex, France \\ bonnet@athena.polytechnique.fr, (1)-69333026 (fax)
}

${ }^{1}$ Eng. Anal with Bound. Elem., 15, 93-102 (1995) 


\section{Introduction and motivation.}

Although variational Galerkin-type boundary integral equation (GBIE) formulations have received a lot of attention in the past, mostly in the fielf of applied mathematics (see e.g. Bendali [2], Cortey-Dymont [8], Nedelec [15], Wendland [22]), developments and applications in the field of solid mechanics (see Sirtori et al. [21], Parreira and Guiggiani [18], Becache [1], Nishimura and Kobayashi [17], Kane and Balakrishna [11]) have been few compared to the very popular collocation boundary integral equation (CBIE) methods.

Variational approaches do have attractive conceptual features compared to usual collocation methods: known convergence results are more extensive [15] and actually better than those of CBEM using the same discretization. They lead to symmetric stiffness-like BEM matrices, so that the solution CPU time, which dominates for large DOF numbers, is asymptotically half that necessary for solving the full nonsymmetric system produced by collocation methods. Indeed the numerical examples in Balakrishna et al. [12] show clearly that, for a given discretization, GBEA becomes computationally more efficient than CBIE for sufficiently large numbers of DOFs.

GBIE formulations can be viewed as weighted residual formulations of CBIEs, and thus usually involve double surface integrals (see however Bui [7], where a symmetric BIE formulation with a single surface integration is discussed). In order to get a symmetric GBIE formulation, weighted residual forms of displacement and traction CBIEs are used respectively on the Dirichlet and Neumann parts of the boundary. Although the collocation traction $\mathrm{BIE}$ is known to require a $C^{1, \alpha}$ continuity for the boundary displacement $\boldsymbol{u}$ at the collocation point, which causes some technical difficulties especially when using curved elements for 3-D situations, its Galerkin counterpart requires only a $C^{0, \alpha}$ continu-

ity for $\boldsymbol{u}$. The usual $C^{0}$ conformal shape functions can then be used without difficulty in a GBEA context.

On the other hand, the implementation of GBIE formulations for general engineering problems is somewhat more involved than that of displacement CBIE, which explains perhaps its relative lack of popularity. Also, investigation of GBIE formulations for mixed boundary value problems began only recently. Another important issue is the handling of singular integrals: the double boundary integrals that appear in Galerkin-type BIE 
formulations are convergent but some of the successive simple integrals are not. One possibility is a proper direct handling of the strongly singular and hypersingular simple

surface integrals that appear, using either a numerical method (Guiggiani et al. [9]) or analytical integration of the singularity (Sirtori et al. [21], Balakrishna et al. [12]; the latter used symbolic computation). Another possibility is to perform, as is done here, an analytical regularization before any discretization.

In the present paper, we investigate the statement of a GBIE formulation for the mixed boundary value problem of linear elastostatics, for either interior or exterior problems, obtained from the stationarity conditions associated to the minimization principle of an augmented potential energy. This variational BIE formulation appears to be precisely that obtained from a weighted residual viewpoint. Also, the present formulation is regularized in the spirit of previous works on indirect regularization of displacement CBIEs [4], [5], [6] and of traction BIEs ([17]). The resulting variational BIE formulation is expressed in terms of weakly singular inner surface integrals followed by outer regular integrals. Also, it is a direct formulation, whose unknowns are the physical displacements and tractions on the boundary. As a by-product, the indirect variational BIE formulation for mixed problems, whose unknowns are fictitious densities, is also established from the direct one. Finally, some implementation features are discussed.

\section{Elastic problem, potential energy and stationarity conditions}

Let $\Omega$ denote a three-dimensional elastic body, either bounded or unbounded; $\boldsymbol{n}$ denotes the unit normal vector outwards from $\Omega$. The elastic displacement $\boldsymbol{u}(\boldsymbol{y})(\boldsymbol{y} \in \Omega)$ is governed by the homogeneous Navier equation:

$$
C_{i j a b} u_{a, b j}=0
$$

where $\boldsymbol{C}$ denotes the Hooke tensor of elastic constants. The comma indicates partial differentiation; Einstein summation convention is used throughout. Besides, mixed boundary 
conditions are considered:

$$
\left\{\begin{aligned}
\boldsymbol{u} & =\boldsymbol{u}^{D} & & \text { on } S_{u} \\
\boldsymbol{T}^{n}(\boldsymbol{u}) & =\boldsymbol{t}^{D} & & \text { on } S_{T}
\end{aligned}\right.
$$

where $S_{u}, S_{T}=\partial \Omega-S_{u}$ are complementary portions of $\partial \Omega$ and

$$
\boldsymbol{T}^{n}(\boldsymbol{u})=[\boldsymbol{C}: \boldsymbol{\varepsilon}(\boldsymbol{u})] . \boldsymbol{n}
$$

is the elastic traction vector associated with $\boldsymbol{u}$. If $\Omega$ is unbounded, suitable decay conditions at infinity must be added to (2).

The solution $\boldsymbol{u}$ to the elastic problem (1-2) minimizes the following augmented potential energy functional $E(\boldsymbol{v})$ :

$$
E(\boldsymbol{v})=\int_{\Omega} \boldsymbol{\varepsilon}(\boldsymbol{v}): C: \boldsymbol{\varepsilon}(\boldsymbol{v}) \mathrm{d} V-\int_{S_{T}} \boldsymbol{t}^{D} \cdot \boldsymbol{v} \mathrm{d} S-\int_{S_{u}} \boldsymbol{t} .\left(\boldsymbol{v}-\boldsymbol{u}^{D}\right) \mathrm{d} S
$$

The last integral term in (3) has the effect that $\boldsymbol{u}$ is an unconstrained minimizer of $E(\boldsymbol{v})$ : no kinematical constraints are imposed on $\boldsymbol{v}$. Thus $\boldsymbol{u}$ can be sought such that the first variation of $E$ vanishes at $\boldsymbol{v}=\boldsymbol{u}$, i.e., letting $\boldsymbol{v}=\boldsymbol{u}+\delta \boldsymbol{u}$, ve have:

$$
\delta E(\boldsymbol{u}) . \delta \boldsymbol{u}=\int_{\Omega} \boldsymbol{\varepsilon}(\boldsymbol{u}): C: \boldsymbol{\varepsilon}(\delta \boldsymbol{u}) \mathrm{d} V-\int_{S_{T}} \boldsymbol{t}^{D} . \delta \boldsymbol{u} \mathrm{d} S-\int_{S_{u}} \boldsymbol{t} . \delta \boldsymbol{u} \mathrm{d} S=0
$$

with no kinematical constraint on $\delta \boldsymbol{u}$. Indeed, from

$$
\int_{\Omega} \boldsymbol{\varepsilon}(\boldsymbol{u}): C: \boldsymbol{\varepsilon}(\delta \boldsymbol{u}) \mathrm{d} V=\int_{\partial \Omega} \boldsymbol{T}^{n}(\boldsymbol{u}) . \delta \boldsymbol{u} \mathrm{d} S-\int_{\Omega} \operatorname{div}[C: \boldsymbol{\varepsilon}(\boldsymbol{u})] . \delta \boldsymbol{u} \mathrm{d} V
$$

it is readily shown that $\boldsymbol{u}$ solves (1-2) and that the Lagrange multiplier $\boldsymbol{t}$ equals the traction vector $\boldsymbol{T}^{n}(\boldsymbol{u})$ on $S_{u}$.

Now let us suppose that the minimization of $E(\boldsymbol{v})$ is attempted only for those $\boldsymbol{v}$ which satisfy the local equilibrium equation (1). Since this is also satisfied by the solution $\boldsymbol{u}$, one is then led to restrict (4) to the trial functions $\delta \boldsymbol{u}=\boldsymbol{v}-\boldsymbol{u}$ which themselves satisfy (1). Upon integration by parts of the domain integral, the stationarity condition (4) thus takes the form

$$
\begin{aligned}
\delta E(\boldsymbol{u}) . \delta \boldsymbol{u}= & \int_{S_{u}} \boldsymbol{u}^{D} \cdot \boldsymbol{T}^{n}(\boldsymbol{\delta} \boldsymbol{u}) \mathrm{d} S+\int_{S_{T}} \boldsymbol{u} \cdot \boldsymbol{T}^{n}(\boldsymbol{\delta} \boldsymbol{u}) \mathrm{d} S \\
& -\int_{S_{T}} \boldsymbol{t}^{D} . \delta \boldsymbol{u} \mathrm{d} S-\int_{S_{u}} \boldsymbol{t} . \delta \boldsymbol{u} \mathrm{d} S=0
\end{aligned}
$$

which constitutes the starting point for the derivation of a direct variational BIE formulation. 


\section{Test functions}

The next important step is the actual construction of test functions $\delta \boldsymbol{u}$ that satisfy (1). In order to do so, recall that any such $\delta \boldsymbol{u}$ admits the integral representation $(\boldsymbol{x}$ interior to $\Omega)$ :

$$
\delta u_{k}(\boldsymbol{x})=-\int_{\partial \Omega} \delta u_{i}(\boldsymbol{y}) n_{j}(\boldsymbol{y}) \Sigma_{i j}^{k}(\boldsymbol{x}, \boldsymbol{y}) \mathrm{d} S_{y}+\int_{\partial \Omega} \delta t_{i}(y) U_{i}^{k}(\boldsymbol{x}, \boldsymbol{y}) \mathrm{d} S_{y}
$$

where $\delta t$ is the traction vector $\boldsymbol{T}^{n}(\boldsymbol{\delta} \boldsymbol{u})$. Similarly, any $\delta \boldsymbol{u}^{+}$which solves (1) in the exterior domain $\Omega^{+}=\mathbb{R}^{3}-\Omega$ satisfies at any $\boldsymbol{x}$ interior to $\Omega$ the complementary (with reference to $\Omega^{+}$) integral representation:

$$
0=-\int_{\partial \Omega} \delta u_{i}^{+}(\boldsymbol{y}) n_{j}(\boldsymbol{y}) \Sigma_{i j}^{k}(\boldsymbol{x}, \boldsymbol{y}) \mathrm{d} S_{y}+\int_{\partial \Omega} \delta t_{i}^{+}(y) U_{i}^{k}(\boldsymbol{x}, \boldsymbol{y}) \mathrm{d} S_{y}
$$

where $\delta \boldsymbol{t}^{+}$stands for $\boldsymbol{T}^{n}\left(\boldsymbol{\delta} \boldsymbol{u}^{+}\right)$. The normal $\boldsymbol{n}$ outwards from $\Omega$ is used in both (6) and (7). The symbols $U_{i}^{k}(\boldsymbol{x}, \boldsymbol{y}), \Sigma_{i j}^{k}(\boldsymbol{x}, \boldsymbol{y})$ denote the $i$ - and $i j$-components of the elastic displacement and stress fields created at $\boldsymbol{y}$ by a unit point force applied at $\boldsymbol{x}$ along the $k$-direction (elastostatic fundamental solution). The full space (Kelvin) solution, a half space solution with a free-surface condition (Mindlin), or any other fundamental solution defined on a subset of $\mathbb{R}^{3}$ that includes $\Omega$ may be used for this purpose. Any of them has the symmetry property:

$$
U_{k}^{a}(\boldsymbol{x}, \boldsymbol{y})=U_{a}^{k}(\boldsymbol{y}, \boldsymbol{x})
$$

which in turn implies:

$$
C_{i j a b} \frac{\partial}{\partial x_{b}} U_{a}^{k}(\boldsymbol{x}, \boldsymbol{y})=\Sigma_{i j}^{a}(\boldsymbol{y}, \boldsymbol{x}) \quad C_{i j a b} \frac{\partial}{\partial x_{b}} \Sigma_{k l}^{a}(\boldsymbol{x}, \boldsymbol{y})=C_{k \ell a b} \frac{\partial}{\partial y_{b}} \Sigma_{i j}^{a}(\boldsymbol{y}, \boldsymbol{x})
$$

Next, (7) is subtracted from (6), giving:

$$
\delta u_{k}(\boldsymbol{x})=\int_{\partial \Omega} \tilde{u}_{i}(\boldsymbol{y}) n_{j}(\boldsymbol{y}) \Sigma_{i j}^{k}(\boldsymbol{x}, \boldsymbol{y}) \mathrm{d} S_{y}-\int_{\partial \Omega} \tilde{t}_{i}(y) U_{i}^{k}(\boldsymbol{x}, \boldsymbol{y}) \mathrm{d} S_{y}
$$

using the displacement and traction jumps accross $\partial \Omega$ : $\tilde{\boldsymbol{u}}=\delta \boldsymbol{u}^{+}-\delta \boldsymbol{u}$ and $\tilde{\boldsymbol{t}}=\delta \boldsymbol{t}^{+}-\delta \boldsymbol{t}$. It is indeed legitimate to restrict the above formula by imposing $\delta \boldsymbol{u}^{+}=\delta \boldsymbol{u}$, i.e. $\tilde{\boldsymbol{u}}=\mathbf{0}$ on $S_{u}$ and $\delta \boldsymbol{t}^{+}=-\delta \boldsymbol{t}$, i.e. $\tilde{\boldsymbol{t}}=\mathbf{0}$ on $S_{T}$, which gives:

$$
\begin{aligned}
\delta u_{k}(\boldsymbol{x}) & =\int_{S_{T}} \tilde{u}_{i}(\boldsymbol{y}) n_{j}(\boldsymbol{y}) \Sigma_{i j}^{k}(\boldsymbol{x}, \boldsymbol{y}) \mathrm{d} S_{y}-\int_{S_{u}} \tilde{t}_{i}(y) U_{i}^{k}(\boldsymbol{x}, \boldsymbol{y}) \mathrm{d} S_{y} \\
& =\delta u_{k}^{T}(\boldsymbol{x})+\delta u_{k}^{U}(\boldsymbol{x})
\end{aligned}
$$


Such test functions $\delta \boldsymbol{u}$ satisfy (1) and reflect the boundary condition structure of the initial mixed elastostatic problem under study. Note that, since $\tilde{\boldsymbol{u}}$ must be continuous all over $\partial \Omega$, one has:

$$
\left.\tilde{\boldsymbol{u}}\right|_{\partial \tilde{S}_{T}}=\mathbf{0}
$$

The stress tensor $\delta \boldsymbol{\sigma}=\boldsymbol{\sigma}(\delta \boldsymbol{u})$ is then given from (10) by the representation:

$$
\delta \sigma_{i j}(\boldsymbol{x})=C_{k l a b} \int_{S_{T}} \tilde{u}_{k}(\boldsymbol{y}) n_{\ell}(\boldsymbol{y}) \frac{\partial}{\partial y_{b}} \Sigma_{i j}^{a}(\boldsymbol{y}, \boldsymbol{x}) \mathrm{d} S_{y}-\int_{S_{u}} \tilde{t}_{k}(y) \Sigma_{i j}^{k}(\boldsymbol{y}, \boldsymbol{x}) \mathrm{d} S_{y}
$$

where use has been made of (8), (9).

\section{Derivation of the direct symmetric bie formulation}

Basically the symmetric BIE formulation comes from the substitution of (10), (13) into the stationarity equation (5). This leads to a formulation in terms of double surface integrals. However, integrability problems, related to the presence of kernels behaving like $r^{-1}, r^{-2}, r^{-3}$ where $r=|\boldsymbol{y}-\boldsymbol{x}|$ (especially the last two), require a limiting process of some kind.

\subsection{Introduction of an auxiliary surface}

Let $\tilde{S}$ be a closed, regular surface exterior to $\Omega$ (hence exterior to $\partial \Omega$ for interior problems, but interior to $\partial \Omega$ for exterior problems), defined by means of a one-to-one mapping $\boldsymbol{F}$ onto $\partial \Omega$ :

$$
\boldsymbol{y} \in \partial \Omega \rightarrow \boldsymbol{z}=\boldsymbol{F}(\boldsymbol{y}) \in \tilde{S}
$$

which is left unspecified. This surface $\tilde{S}$ is substituted to $\partial \Omega$ in (10), (13), following an approach used by Sirtori et al. [21]. The idea is to perform some analytical manipulations, for regularization purposes, on the double surface integrals over $\partial \Omega \times \tilde{S}$ (in which case the elastic kernels are nonsingular) and then consider the limiting process $\tilde{S} \rightarrow \partial \Omega$.

The test functions $\delta \boldsymbol{u}, \delta \boldsymbol{\sigma}$ are thus given by:

$$
\begin{aligned}
\delta u_{k}(\boldsymbol{x}) & =\int_{\tilde{S}_{T}} \tilde{u}_{i}(\boldsymbol{y}) n_{j}(\boldsymbol{y}) \Sigma_{i j}^{k}(\boldsymbol{x}, \boldsymbol{y}) \mathrm{d} S_{y}-\int_{\tilde{S}_{u}} \tilde{t}_{i}(y) U_{i}^{k}(\boldsymbol{x}, \boldsymbol{y}) \mathrm{d} S_{y} \\
\delta \sigma_{i j}(\boldsymbol{x}) & =C_{k l a b} \int_{\tilde{S}_{T}} \tilde{u}_{k}(\boldsymbol{y}) n_{\ell}(\boldsymbol{y}) \frac{\partial}{\partial y_{b}} \Sigma_{i j}^{a}(\boldsymbol{y}, \boldsymbol{x}) \mathrm{d} S_{y}-\int_{\tilde{S}_{u}} \tilde{t}_{k}(y) \Sigma_{i j}^{k}(\boldsymbol{y}, \boldsymbol{x}) \mathrm{d} S_{y}
\end{aligned}
$$




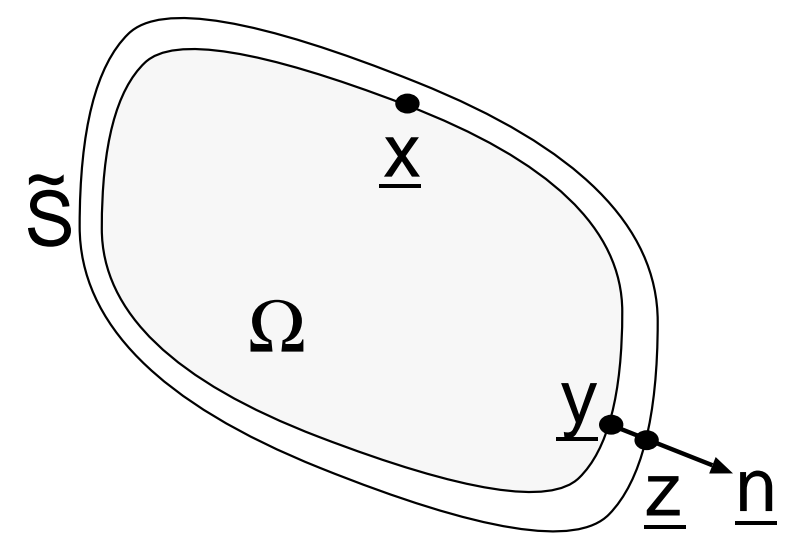

Figure 1: Boundary $\partial \Omega$ and auxiliary surface $\tilde{S}$

(with $\tilde{S}_{u}=\boldsymbol{F}\left(S_{u}\right), \tilde{S}_{T}=\boldsymbol{F}\left(S_{T}\right)$ ). Recall that eqns. (14), (15) apply for $\Omega$ either bounded or unbounded, $\boldsymbol{n}$ being the unit normal outwards from $\Omega$.

Upon substitution of (14), (15), the stationarity condition (5) for $E(\boldsymbol{u})$ can be split into two independent parts:

$$
\begin{cases}\forall \tilde{\boldsymbol{u}} & \delta E^{T}=0 \\ \forall \tilde{\boldsymbol{t}} & \delta E^{U}=0\end{cases}
$$

where $\delta E^{T}$ and $\delta E^{U}$ collect the terms containing $\tilde{\boldsymbol{u}}$ and $\tilde{\boldsymbol{t}}$ respectively that arise from the substitution of (14), (15) into (5).

\subsection{Evaluation of $\delta E^{U}$}

From eqns (5), (14), (15), one has:

$$
\begin{aligned}
\delta E^{U}= & I_{1}+I_{2} \\
I_{1}= & \int_{S_{u}} t_{k}(\boldsymbol{x}) \int_{\tilde{S}_{u}} \tilde{t}_{i}(\boldsymbol{z}) U_{i}^{k}(\boldsymbol{x}, \boldsymbol{z}) \mathrm{d} S_{z} \mathrm{~d} S_{x} \\
& +\int_{S_{T}} t_{k}^{D}(\boldsymbol{x}) \int_{\tilde{S}_{u}} \tilde{t}_{i}(\boldsymbol{z}) U_{i}^{k}(\boldsymbol{x}, \boldsymbol{z}) \mathrm{d} S_{z} \mathrm{~d} S_{x} \\
I_{2}= & -\int_{S_{u}} u_{i}^{D}(\boldsymbol{x}) n_{j}(\boldsymbol{x}) \int_{\tilde{S}_{u}} \tilde{t}_{k}(\boldsymbol{z}) \Sigma_{i j}^{k}(\boldsymbol{z}, \boldsymbol{x}) \mathrm{d} S_{z} \mathrm{~d} S_{x} \\
& -\int_{S_{T}} u_{i}(\boldsymbol{x}) n_{j}(\boldsymbol{x}) \int_{\tilde{S}_{u}} \tilde{t}_{k}(\boldsymbol{z}) \sum_{i j}^{k}(\boldsymbol{z}, \boldsymbol{x}) \mathrm{d} S_{z} \mathrm{~d} S_{x}
\end{aligned}
$$

First, the term $I_{1}$ is made of a (potentially) weakly singular surface integral followed by a regular one and thus needs no regularization; it is left unchanged. 
Then, $I_{2}$, eqn. (19), is rewritten as:

$$
\begin{aligned}
I_{2}= & -\int_{\tilde{S}_{u}} \tilde{t}_{k}(\boldsymbol{z}) \int_{S_{u}}\left[u_{i}^{D}(\boldsymbol{x})-u_{i}^{D}(\boldsymbol{y})\right] n_{j}(\boldsymbol{x}) \sum_{i j}^{k}(\boldsymbol{z}, \boldsymbol{x}) \mathrm{d} S_{x} \mathrm{~d} S_{z} \\
& -\int_{\tilde{S}_{u}} \tilde{t}_{k}(\boldsymbol{z}) \int_{S_{T}}\left[u_{i}(\boldsymbol{x})-u_{i}^{D}(\boldsymbol{y})\right] n_{j}(\boldsymbol{x}) \Sigma_{i j}^{k}(\boldsymbol{z}, \boldsymbol{x}) \mathrm{d} S_{x} \mathrm{~d} S_{z} \\
& -\int_{\tilde{S}_{u}} \tilde{t}_{k}(\boldsymbol{z}) u_{i}^{D}(\boldsymbol{y}) \int_{\partial \Omega} n_{j}(\boldsymbol{x}) \Sigma_{i j}^{k}(\boldsymbol{z}, \boldsymbol{x}) \mathrm{d} S_{x} \mathrm{~d} S_{z}
\end{aligned}
$$

where $\boldsymbol{y} \in S_{u}$ is the point such that $\boldsymbol{F}(\boldsymbol{y})=\boldsymbol{z} \in \tilde{S}_{u}$ (hence $\boldsymbol{u}(\boldsymbol{y})=\boldsymbol{u}^{D}(\boldsymbol{y})$ ). Integrations over $\partial \Omega$ and $\tilde{S}$ have been interchanged, this operation being valid because of the current nonsingular character of the integrals.

Besides, one notes that the fundamental stress tensor satisfies, by virtue of equilibrium, the following identity (the source point $\boldsymbol{z}$ being exterior to $\Omega$ ):

$$
\int_{\partial \Omega} n_{j}(\boldsymbol{x}) \sum_{i j}^{k}(\boldsymbol{z}, \boldsymbol{x}) \mathrm{d} S_{x}=\kappa \delta_{i k}
$$

where $\kappa=0$ ( $\Omega$ bounded, $\boldsymbol{n}$ exterior to $\partial \Omega)$ or $\kappa=1$ ( $\mathbb{R}^{3}-\Omega$ bounded, $\boldsymbol{n}$ interior to $\partial \Omega$ ). Thus, the last integral in (20) becomes:

$$
\int_{\tilde{S}_{u}} \tilde{t}_{k}(\boldsymbol{z}) u_{i}^{D}(\boldsymbol{y}) \int_{\partial \Omega} n_{j}(\boldsymbol{x}) \Sigma_{i j}^{k}(\boldsymbol{z}, \boldsymbol{x}) \mathrm{d} S_{x} \mathrm{~d} S_{z}=\kappa \int_{\tilde{S}_{u}} \tilde{t}_{k}(\boldsymbol{z}) u_{k}^{D}(\boldsymbol{y}) \mathrm{d} S_{z}
$$

These manipulations result in a regularization of the initially strongly singular integral w.r.t. $\boldsymbol{x}$ in (19). Indeed one notes that, since

$$
\boldsymbol{U}(\boldsymbol{z}, \boldsymbol{x}) \sim|\boldsymbol{z}-\boldsymbol{x}|^{-1} \quad \boldsymbol{\Sigma}(\boldsymbol{z}, \boldsymbol{x}) \sim|\boldsymbol{z}-\boldsymbol{x}|^{-2}
$$

the expressions (18), (20) involve weakly singular integrals w.r.t. $\boldsymbol{x}$ followed by nonsingular integrals w.r.t. $\boldsymbol{z}$, provided the regularity requirement $\boldsymbol{u} \in C^{0, \alpha}(\partial \Omega)$, i.e.:

$$
\exists C>0, \exists \alpha \in] 0,1] \quad|\boldsymbol{u}(\boldsymbol{y})-\boldsymbol{u}(\boldsymbol{x})| \leq C|\boldsymbol{y}-\boldsymbol{x}|^{\alpha}
$$

is met. Then, the limiting expression of $I_{2}, I_{1}$ for $\tilde{S} \rightarrow \partial \Omega$ is obtained by a mere substi- 
tution of $\left(\tilde{S}_{u}, \boldsymbol{z}\right)$ by $\left(S_{u}, \boldsymbol{y}\right)$ in eqns. (18), (20). The result is thus:

$$
\begin{aligned}
I_{1}= & \int_{S_{u}} t_{k}(\boldsymbol{x}) \int_{S_{u}} \tilde{t}_{i}(\boldsymbol{y}) U_{i}^{k}(\boldsymbol{x}, \boldsymbol{y}) \mathrm{d} S_{y} \mathrm{~d} S_{x} \\
& +\int_{S_{T}} t_{k}^{D}(\boldsymbol{x}) \int_{S_{u}} \tilde{t}_{i}(\boldsymbol{y}) U_{i}^{k}(\boldsymbol{x}, \boldsymbol{y}) \mathrm{d} S_{y} \mathrm{~d} S_{x} \\
I_{2}= & -\int_{S_{u}} \tilde{t}_{k}(\boldsymbol{y}) \int_{S_{u}}\left[u_{i}^{D}(\boldsymbol{x})-u_{i}^{D}(\boldsymbol{y})\right] n_{j}(\boldsymbol{x}) \Sigma_{i j}^{k}(\boldsymbol{y}, \boldsymbol{x}) \mathrm{d} S_{x} \mathrm{~d} S_{y} \\
& -\int_{S_{u}} \tilde{t}_{k}(\boldsymbol{y}) \int_{S_{T}}\left[u_{i}(\boldsymbol{x})-u_{i}^{D}(\boldsymbol{y})\right] n_{j}(\boldsymbol{x}) \Sigma_{i j}^{k}(\boldsymbol{y}, \boldsymbol{x}) \mathrm{d} S_{x} \mathrm{~d} S_{y} \\
& -\kappa \int_{\tilde{S}_{u}} \tilde{t}_{k}(\boldsymbol{z}) u_{k}^{D}(\boldsymbol{y}) \mathrm{d} S_{y}
\end{aligned}
$$

For eqn. (25) above, use has been made of the substitution of (21) into (19).

\subsection{Evaluation of $\delta E^{T}$}

From eqns (5), (14), (15), one has:

$$
\begin{aligned}
\delta E^{T}= & J_{1}+J_{2} \\
J_{1}= & -\int_{S_{u}} t_{k}(\boldsymbol{x}) \int_{\tilde{S}_{T}} \tilde{u}_{i}(\boldsymbol{z}) n_{j}(\boldsymbol{z}) \Sigma_{i j}^{k}(\boldsymbol{x}, \boldsymbol{z}) \mathrm{d} S_{z} \mathrm{~d} S_{x} \\
& -\int_{S_{T}} t_{k}^{D}(\boldsymbol{x}) \int_{\tilde{S}_{T}} \tilde{u}_{i}(\boldsymbol{z}) n_{j}(\boldsymbol{z}) \Sigma_{i j}^{k}(\boldsymbol{x}, \boldsymbol{z}) \mathrm{d} S_{z} \mathrm{~d} S_{x} \\
J_{2}= & \int_{S_{u}} u_{i}^{D}(\boldsymbol{x}) n_{j}(\boldsymbol{x}) C_{k l a b} \int_{\tilde{S}_{T}} \tilde{u}_{k}(\boldsymbol{z}) n_{\ell}(\boldsymbol{z}) \frac{\partial}{\partial z_{b}} \Sigma_{i j}^{a}(\boldsymbol{z}, \boldsymbol{x}) \mathrm{d} S_{z} \mathrm{~d} S_{x} \\
& +\int_{S_{T}} u_{i}(\boldsymbol{x}) n_{j}(\boldsymbol{x}) C_{k l a b} \int_{\tilde{S}_{T}} \tilde{u}_{k}(\boldsymbol{z}) n_{\ell}(\boldsymbol{z}) \frac{\partial}{\partial z_{b}} \Sigma_{i j}^{a}(\boldsymbol{z}, \boldsymbol{x}) \mathrm{d} S_{z} \mathrm{~d} S_{x}
\end{aligned}
$$

First, $J_{1}$ is subjected to a treatment similar to that made to $I_{2}$. For this purpose, eqn. (27) is rewritten, upon addition and subtraction of $\tilde{\boldsymbol{u}}(\boldsymbol{x})$ to $\tilde{\boldsymbol{u}}(\boldsymbol{z})$, as:

$$
\begin{aligned}
J_{1}= & -\int_{S_{u}} t_{k}(\boldsymbol{x}) \int_{\tilde{S}_{T}} \tilde{u}_{i}(\boldsymbol{z}) n_{j}(\boldsymbol{z}) \Sigma_{i j}^{k}(\boldsymbol{x}, \boldsymbol{z}) \mathrm{d} S_{z} \mathrm{~d} S_{x} \\
& -\int_{S_{T}} t_{k}^{D}(\boldsymbol{x}) \int_{\tilde{S}_{T}}\left[\tilde{u}_{i}(\boldsymbol{z})-\tilde{u}_{i}(\boldsymbol{x})\right] n_{j}(\boldsymbol{z}) \Sigma_{i j}^{k}(\boldsymbol{x}, \boldsymbol{z}) \mathrm{d} S_{z} \mathrm{~d} S_{x} \\
& -\int_{S_{T}} t_{k}^{D}(\boldsymbol{x}) \tilde{u}_{i}(\boldsymbol{x}) \int_{\tilde{S}_{T}} n_{j}(\boldsymbol{z}) \Sigma_{i j}^{k}(\boldsymbol{x}, \boldsymbol{z}) \mathrm{d} S_{z} \mathrm{~d} S_{x}
\end{aligned}
$$

where the fact that $\tilde{\boldsymbol{u}}=\mathbf{0}$ for $\boldsymbol{x} \in S_{u}$ has been used. Besides, the fundamental stress tensor satisfies the following identity, similar to (21) but with the source point $\boldsymbol{x}$ now being interior to $\Omega$ :

$$
\int_{\tilde{S}} n_{j}(\boldsymbol{z}) \Sigma_{i j}^{k}(\boldsymbol{x}, \boldsymbol{z}) \mathrm{d} S_{z}=(\kappa-1) \delta_{i k}
$$


where again $\kappa=0$ ( $\Omega$ bounded, $\boldsymbol{n}$ exterior to $\partial \Omega$ ) or $\kappa=1\left(\mathbb{R}^{3}-\Omega\right.$ bounded, $\boldsymbol{n}$ interior to $\partial \Omega$ ). The last integral in (29) then becomes:

$$
\begin{aligned}
& \int_{S_{T}} t_{k}^{D}(\boldsymbol{x}) \tilde{u}_{i}(\boldsymbol{x}) \int_{\tilde{S}_{u}} n_{j}(\boldsymbol{z}) \Sigma_{i j}^{k}(\boldsymbol{x}, \boldsymbol{z}) \mathrm{d} S_{z} \mathrm{~d} S_{x} \\
& =\int_{S_{T}} t_{k}^{D}(\boldsymbol{x}) \tilde{u}_{i}(\boldsymbol{x})\left\{(\kappa-1) \delta_{i k}-\int_{\tilde{S}_{u}} n_{j}(\boldsymbol{z}) \Sigma_{i j}^{k}(\boldsymbol{x}, \boldsymbol{z}) \mathrm{d} S_{z}\right\} \mathrm{d} S_{x} \\
& =(\kappa-1) \int_{S_{T}} t_{k}^{D}(\boldsymbol{x}) \tilde{u}_{k}(\boldsymbol{x}) \mathrm{d} S_{x}-\int_{S_{T}} t_{k}^{D}(\boldsymbol{x}) \tilde{u}_{i}(\boldsymbol{x}) \int_{\tilde{S}_{u}} n_{j}(\boldsymbol{z}) \Sigma_{i j}^{k}(\boldsymbol{x}, \boldsymbol{z}) \mathrm{d} S_{z} \mathrm{~d} S_{x}
\end{aligned}
$$

Substitution of (31) into (29) allows for an expression of $J_{1}$ which is made, in the limiting case $\tilde{S} \rightarrow \partial \Omega$, of weakly singular integrals over $S_{T}$ followed by nonsingular integrals over $S_{u}, S_{T}$, as follows:

$$
\begin{aligned}
J_{1}= & -\int_{S_{u}} t_{k}(\boldsymbol{x}) \int_{S_{T}} \tilde{u}_{i}(\boldsymbol{y}) n_{j}(\boldsymbol{y}) \Sigma_{i j}^{k}(\boldsymbol{x}, \boldsymbol{y}) \mathrm{d} S_{y} \mathrm{~d} S_{x} \\
& -\int_{S_{T}} t_{k}^{D}(\boldsymbol{x}) \int_{S_{T}}\left[\tilde{u}_{i}(\boldsymbol{y})-\tilde{u}_{i}(\boldsymbol{x})\right] n_{j}(\boldsymbol{y}) \Sigma_{i j}^{k}(\boldsymbol{x}, \boldsymbol{y}) \mathrm{d} S_{y} \mathrm{~d} S_{x} \\
& +(1-\kappa) \int_{S_{T}} t_{k}^{D}(\boldsymbol{x}) \tilde{u}_{k}(\boldsymbol{x}) \mathrm{d} S_{x} \\
& +\int_{S_{T}} t_{k}(\boldsymbol{x}) \tilde{u}_{i}(\boldsymbol{x}) \int_{S_{u}} n_{j}(\boldsymbol{y}) \Sigma_{i j}^{k}(\boldsymbol{x}, \boldsymbol{y}) \mathrm{d} S_{y} \mathrm{~d} S_{x}
\end{aligned}
$$

The weakly singular character of the inner integral in the above equation relies upon $\tilde{\boldsymbol{u}}$ being $C^{0, \alpha}$-continuous over $\partial \Omega$, eqn. (23). The property (12) of $\tilde{\boldsymbol{u}}$, together with (23), implies:

$$
\exists C>0, \exists \alpha \in] 0,1] \quad|\boldsymbol{u}(\boldsymbol{y})| \leq C|\boldsymbol{y}-\boldsymbol{x}| \quad \boldsymbol{x} \in S_{u}, \boldsymbol{y} \in S_{T}
$$

and thus also contributes to the weakly singular character of (32):

Next, let us consider the second integral $J_{2}$ in (26), which involves the hypersingular kernel $C_{k l a b} \frac{\partial}{\partial z_{b}} \sum_{i j}^{a}(\boldsymbol{z}, \boldsymbol{x})$. Following an argument given by Nishimura and Kobayashi [16] and using the symmetry property (9), one notes that, for $\boldsymbol{x} \neq \boldsymbol{y}$ :

$$
\begin{aligned}
& \frac{\partial}{\partial x_{j}}\left\{C_{k l a b} \frac{\partial}{\partial x_{b}} \Sigma_{i j}^{a}(\boldsymbol{z}, \boldsymbol{x})\right\}=C_{k l a b} \frac{\partial}{\partial x_{b}}\left\{\frac{\partial}{\partial x_{j}} \Sigma_{i j}^{a}(\boldsymbol{z}, \boldsymbol{x})\right\}=0 \\
& \frac{\partial}{\partial z_{l}}\left\{C_{k l a b} \frac{\partial}{\partial z_{b}} \Sigma_{i j}^{a}(\boldsymbol{z}, \boldsymbol{x})\right\}=C_{i j a b} \frac{\partial}{\partial x_{b}}\left\{\frac{\partial}{\partial z_{l}} \Sigma_{k l}^{a}(\boldsymbol{x}, \boldsymbol{z})\right\}=0
\end{aligned}
$$

This implies the existence of a fourth-order tensor $\boldsymbol{A}$ such that:

$$
C_{k l a b} \frac{\partial}{\partial z_{b}} \Sigma_{i j}^{a}(\boldsymbol{z}, \boldsymbol{x})=e_{i e p} e_{j f q} e_{k g r} e_{l h s} \frac{\partial}{\partial x_{e}} \frac{\partial}{\partial x_{f}} \frac{\partial}{\partial z_{g}} \frac{\partial}{\partial z_{h}} A_{p q r s}(\boldsymbol{z}, \boldsymbol{x}) \quad(\boldsymbol{z} \neq \boldsymbol{x})
$$


where $e_{i j k}$ are the components of the permutation tensor. For instance, the tensor $\boldsymbol{A}(\boldsymbol{z}, \boldsymbol{x})$ associated with the three-dimensional isotropic Kelvin fundamental solution is given (Nedelec [15]) by:

$$
A_{p q r s}(\boldsymbol{z}, \boldsymbol{x})=\frac{\mu}{8 \pi}\left[\delta_{p r} \delta_{q s}+\delta_{p s} \delta_{q r}+\frac{2 \nu}{1-\nu} \delta_{p q} \delta_{r s}\right]|\boldsymbol{z}-\boldsymbol{x}|
$$

The decomposition (33) allows for the use of the following variant of Stokes' formula:

$$
\int_{S} g e_{a b c} n_{a} f_{, b} \mathrm{~d} S=-\int_{S} f e_{a b c} n_{a} g_{, b} \mathrm{~d} S
$$

where $S$ is any regular surface; moreover either $S$ is closed or $f=0$ on $\partial S$. Here it is applied twice (once w.r.t. $\boldsymbol{z}$ and once w.r.t. $\boldsymbol{x}$ ) to $J_{2}$, eqn. (28); this gives:

$$
\begin{aligned}
J_{2}= & \int_{S_{u}}\left(R u^{D}\right)_{i q}(\boldsymbol{x}) \int_{\tilde{S}_{T}}(R \tilde{u})_{k s}(\boldsymbol{z}) B_{i k q s}(\boldsymbol{z}, \boldsymbol{x}) \mathrm{d} S_{z} \mathrm{~d} S_{x} \\
& +\int_{S_{T}}(R u)_{i q}(\boldsymbol{x}) \int_{\tilde{S}_{T}}(R \tilde{u})_{k s}(\boldsymbol{z}) B_{i k q s}(\boldsymbol{z}, \boldsymbol{x}) \mathrm{d} S_{z} \mathrm{~d} S_{x}
\end{aligned}
$$

using the notations

$$
\begin{aligned}
(R u)_{i q} & =e_{j f q} n_{j} u_{i, f} \\
B_{i k q s}(\boldsymbol{z}, \boldsymbol{x}) & =e_{i e p} e_{k g r} \frac{\partial}{\partial x_{e}} \frac{\partial}{\partial z_{g}} A_{p q r s}(\boldsymbol{z}, \boldsymbol{x})
\end{aligned}
$$

The result (35) makes use of the property (12) of $\tilde{\boldsymbol{u}}$. One notes that

$$
\boldsymbol{B}(\boldsymbol{z}, \boldsymbol{x}) \sim|\boldsymbol{z}-\boldsymbol{x}|^{-1}
$$

which stems from (33), (37). This implies that eqn. (35) involves a weakly singular inner (w.r.t. $\boldsymbol{x}$ ) integral followed by a nonsingular outer (w.r.t. $\boldsymbol{z}$ ) integral. The limiting expression of $J_{2}$ for $\tilde{S} \rightarrow \partial \Omega$ is thus obtained by a simple replacement of $\left(\tilde{S}_{T}, \boldsymbol{z}\right)$ with $\left(S_{T}, \boldsymbol{y}\right)$ in (35):

$$
\begin{aligned}
J_{2}= & \int_{S_{u}}\left(R u^{D}\right)_{i q}(\boldsymbol{x}) \int_{S_{T}}(R \tilde{u})_{k s}(\boldsymbol{y}) B_{i k q s}(\boldsymbol{y}, \boldsymbol{x}) \mathrm{d} S_{y} \mathrm{~d} S_{x} \\
& +\int_{S_{T}}(R u)_{i q}(\boldsymbol{x}) \int_{S_{T}}(R \tilde{u})_{k s}(\boldsymbol{y}) B_{i k q s}(\boldsymbol{y}, \boldsymbol{x}) \mathrm{d} S_{y} \mathrm{~d} S_{x}
\end{aligned}
$$

\subsection{Statement of the direct symmetric BIE formulation}

The previous results are now put together. From (16), the definitions (17), (26) and the results $(24),(25),(32),(38)$, the direct symmetric BIE formulation for the mixed elastic 
boundary value problem reads:

Find $\boldsymbol{u} \in \mathcal{V}_{u}, \boldsymbol{t} \in \mathcal{V}_{T}$

$$
\left\{\begin{array}{rl}
\mathcal{B}_{u u}(\boldsymbol{u}, \tilde{\boldsymbol{u}})+\mathcal{B}_{t u}(\boldsymbol{t}, \tilde{\boldsymbol{u}}) & =\mathcal{L}_{u}(\tilde{\boldsymbol{u}}) \\
\mathcal{B}_{u t}(\boldsymbol{u}, \tilde{\boldsymbol{t}})+\mathcal{B}_{t t}(\boldsymbol{t}, \tilde{\boldsymbol{t}}) & =\mathcal{L}_{t}(\tilde{\boldsymbol{t}})
\end{array} \quad \forall \tilde{\boldsymbol{u}} \in \mathcal{V}_{u}^{0}, \forall \tilde{\boldsymbol{t}} \in \mathcal{V}_{T}\right.
$$

using the bilinear forms

$$
\begin{aligned}
\mathcal{B}_{u u}(\boldsymbol{u}, \tilde{\boldsymbol{u}}) & =\int_{S_{T}}(R u)_{i q}(\boldsymbol{x}) \int_{S_{T}} B_{i q k s}(\boldsymbol{y}, \boldsymbol{x})(R \tilde{u})_{k s}(\boldsymbol{y}) \mathrm{d} S_{y} \mathrm{~d} S_{x} \\
\mathcal{B}_{t u}(\boldsymbol{t}, \tilde{\boldsymbol{u}}) & =-\int_{S_{u}} \int_{S_{T}} t_{k}(\boldsymbol{x}) \tilde{u}_{i}(\boldsymbol{y}) n_{j}(\boldsymbol{y}) \sum_{i j}^{k}(\boldsymbol{x}, \boldsymbol{y}) \mathrm{d} S_{y} \mathrm{~d} S_{x} \\
\mathcal{B}_{u t}(\boldsymbol{u}, \tilde{\boldsymbol{t}}) & =-\int_{S_{u}} \int_{S_{T}} \tilde{t}_{k}(\boldsymbol{y})\left[u_{i}(\boldsymbol{x})-u_{i}^{D}(\boldsymbol{y})\right] n_{j}(\boldsymbol{x}) \Sigma_{i j}^{k}(\boldsymbol{y}, \boldsymbol{x}) \mathrm{d} S_{x} \mathrm{~d} S_{y} \\
\mathcal{B}_{t t}(\boldsymbol{u}, \tilde{\boldsymbol{t}}) & =\int_{S_{u}} \int_{S_{u}} t_{k}(\boldsymbol{x}) \tilde{t}_{i}(\boldsymbol{y}) U_{i}^{k}(\boldsymbol{x}, \boldsymbol{y}) \mathrm{d} S_{y} \mathrm{~d} S_{x}
\end{aligned}
$$

and the linear forms

$$
\begin{aligned}
\mathcal{L}_{u}(\tilde{\boldsymbol{u}})= & (\kappa-1) \int_{S_{T}} t_{k}^{D}(\boldsymbol{x}) \tilde{u}_{k}(\boldsymbol{x}) \mathrm{d} S_{x} \\
& +\int_{S_{T}} t_{k}^{D}(\boldsymbol{x}) \int_{S_{T}}\left[\tilde{u}_{i}(\boldsymbol{y})-\tilde{u}_{i}(\boldsymbol{x})\right] n_{j}(\boldsymbol{y}) \Sigma_{i j}^{k}(\boldsymbol{x}, \boldsymbol{y}) \mathrm{d} S_{y} \mathrm{~d} S_{x} \\
& -\int_{S_{T}} t_{k}^{D}(\boldsymbol{x}) \tilde{u}_{i}(\boldsymbol{x}) \int_{S_{u}} n_{j}(\boldsymbol{y}) \Sigma_{i j}^{k}(\boldsymbol{x}, \boldsymbol{y}) \mathrm{d} S_{y} \mathrm{~d} S_{x} \\
& -\int_{S_{T}}\left(R u^{D}\right)_{i q}(\boldsymbol{x}) \int_{S_{u}} B_{i q k s}(\boldsymbol{y}, \boldsymbol{x})(R \tilde{u})_{k s}(\boldsymbol{y}) \mathrm{d} S_{y} \mathrm{~d} S_{x} \\
\mathcal{L}_{t}(\tilde{\boldsymbol{t}})= & \kappa \int_{S_{u}} \tilde{t}_{k}(\boldsymbol{x}) u_{k}^{D}(\boldsymbol{x}) \mathrm{d} S_{x} \\
& +\int_{S_{u}} \tilde{t}_{k}(\boldsymbol{y}) \int_{S_{u}}\left[u_{i}^{D}(\boldsymbol{x})-u_{i}^{D}(\boldsymbol{y})\right] n_{j}(\boldsymbol{x}) \Sigma_{i j}^{k}(\boldsymbol{y}, \boldsymbol{x}) \mathrm{d} S_{x} \mathrm{~d} S_{y} \\
& -\int_{S_{u}} \int_{S_{T}} t_{k}^{D}(\boldsymbol{x}) \tilde{t}_{i}(\boldsymbol{y}) U_{i}^{k}(\boldsymbol{x}, \boldsymbol{y}) \mathrm{d} S_{y} \mathrm{~d} S_{x}
\end{aligned}
$$

and where the spaces of admissible functions $\mathcal{V}_{u}, \mathcal{V}_{u}^{0}, \mathcal{V}_{T}$ are defined as:

$$
\begin{aligned}
\mathcal{V}_{u} & =\left\{\boldsymbol{u} \mid \boldsymbol{u} \in C^{0, \alpha}\left(S_{T}\right) \text { and } \boldsymbol{u}=\boldsymbol{u}^{D} \text { on } \partial S_{T}\right\} \\
\mathcal{V}_{u}^{0} & =\left\{\tilde{\boldsymbol{u}} \mid \tilde{\boldsymbol{u}} \in C^{0, \alpha}\left(S_{T}\right) \text { and } \tilde{\boldsymbol{u}}=\mathbf{0} \text { on } \partial S_{T}\right\} \\
\mathcal{V}_{T} & =\left\{\tilde{\boldsymbol{t}} \mid \tilde{\boldsymbol{t}} \text { piecewise continuous on } S_{T}\right\}
\end{aligned}
$$

Note that, contrary to $(\boldsymbol{u}, \tilde{\boldsymbol{u}})$, no restriction is made on the values of $\boldsymbol{t}, \tilde{\boldsymbol{t}}$ along $\partial S_{T}$.

Eqns. (39) to (45) constitute the main result of this paper. Being the stationarity condition (5) applied to an appropriate family of test functions $\delta \boldsymbol{u}$, they define a variational BIE formulation for the mixed elastostatic boundary-value problem. In addition, 
the formulation is symmetric by virtue of the relations

$$
\forall(\boldsymbol{u}, \tilde{\boldsymbol{u}}) \forall(\boldsymbol{t}, \tilde{\boldsymbol{t}})\left\{\begin{aligned}
\mathcal{B}_{u u}(\boldsymbol{u}, \tilde{\boldsymbol{u}}) & =\mathcal{B}_{u u}(\tilde{\boldsymbol{u}}, \boldsymbol{u}) \\
\mathcal{B}_{t t}(\boldsymbol{t}, \tilde{\boldsymbol{t}}) & =\mathcal{B}_{t t}(\tilde{\boldsymbol{t}}, \boldsymbol{t}) \\
\mathcal{B}_{u t}(\boldsymbol{u}, \tilde{\boldsymbol{t}}) & =\mathcal{B}_{t u}(\tilde{\boldsymbol{t}}, \boldsymbol{u})
\end{aligned}\right.
$$

which are visible consequences of formulae (40), (41), (42), (43). Those symmetry properties may be viewed as consequences of the symmetry properties (8), (9), which are true for any elastostatic fundamental solution.

Moreover, the present variational symmetric BIE formulation is direct, i.e. expressed in terms of the physical elastic boundary unknowns $\left.\boldsymbol{u}\right|_{S_{T}},\left.\boldsymbol{t}\right|_{S_{u}}$. The prescribed displacements $\boldsymbol{u}^{D}$ and tractions $\boldsymbol{t}^{D}$ appear in the linear forms $\mathcal{L}_{u}$ and $\mathcal{L}_{t}$.

Also, the formulation (39 to 45) is valid whatever fundamental solution is used. However, the kernel $\boldsymbol{B}(\boldsymbol{y}, \boldsymbol{x})$ depends on the actual fundamental solution used and is currently known analytically only in a few cases like the Kelvin solution (see eqn. (34) for the isotropic case or Becache [1] for the anisotropic case).

The present formulation is established for elastic domains either bounded or unbounded. They slightly differ from each other by the presence of the coefficient $\kappa$ in the expressions (44), (45) for the linear forms $\mathcal{L}_{u}, \mathcal{L}_{t}$.

Finally, the regularization of the $J_{2}$ term, subsection 4.3, was first proposed by Nedelec [15], for Neumann problems. It is valid provided $\boldsymbol{u}, \tilde{\boldsymbol{u}}$ are $C^{0, \alpha}$-continuous over $\partial \Omega$ and $S_{T}$ respectively. On the other hand, the weighted residual formulation of the traction CBIE requires a $C^{1, \alpha}$ continuity for $\boldsymbol{u}$ at the collocation point no matter which integration technique is used. This is of practical importance since $C^{0, \alpha}$ interpolations are easier to handle and more flexible, especially for 3-D situations.

\subsection{Relation to weighted residual statements}

The displacement and traction exterior representation formulas are respectively:

$$
\begin{aligned}
& 0=\int_{\partial \Omega} u_{i}(\boldsymbol{x}) n_{j}(\boldsymbol{x}) \Sigma_{i j}^{k}(\boldsymbol{z}, \boldsymbol{x}) \mathrm{d} S_{x}-\int_{\partial \Omega} t_{i}(y) U_{i}^{k}(\boldsymbol{z}, \boldsymbol{x}) \mathrm{d} S_{x} \\
& 0=n_{j}(\boldsymbol{z})\left\{C_{i j a b} \int_{\partial \Omega} u_{k}(\boldsymbol{x}) n_{\ell}(\boldsymbol{x}) \frac{\partial}{\partial z_{b}} \Sigma_{k \ell}^{a}(\boldsymbol{z}, \boldsymbol{x}) \mathrm{d} S_{x}-\int_{\partial \Omega} t_{k}(y) \Sigma_{i j}^{k}(\boldsymbol{z}, \boldsymbol{x}) \mathrm{d} S_{x}\right\}
\end{aligned}
$$


where $\boldsymbol{z}$ is any point exterior to $\Omega$ and $\boldsymbol{n}(\boldsymbol{z})$ any unit vector attached to $\boldsymbol{z}$. One then readily sees that equations (17-24-25) are obtained by taking the inner product of eqn. (47) by a fictitious traction $\tilde{t}_{i}(\boldsymbol{z})$, then integrating the result over $\tilde{S}_{T}$. Also, (26-32-38) come from the inner product of eqn. (48) by a fictitious displacement $\tilde{u}_{i}(\boldsymbol{z})$, integrated over $\tilde{S}_{u}$, with $\boldsymbol{n}$ the unit normal to $\tilde{S}_{T}$ pointing away from $\Omega$. The surface $\tilde{S}=\tilde{S}_{u} \cup \tilde{S}_{T}$ is defined as in subsection 4.1 above, and the symmetry properties (8), (9) are used.

As a result, the variational symmetric BIE formulation (39 to 45) is identical to that obtained from a weighted residual statement of displacement and traction collocation BIEs and a regularization approach following the lines of subsections 4.2, 4.3. It is also similar in nature to the weighted residual statement developed in [11], from which it differs mainly by the kind of regularization used for the originally hypersingular kernel.

\subsection{Formulations for Dirichlet amd Neumann problems}

The formulation (39 to 45) is applicable to the special cases of Dirichlet $\left(S_{u}=\partial \Omega, S_{T}=\emptyset\right)$ or Neumann $\left(S_{T}=\partial \Omega, S_{u}=\emptyset\right)$ boundary conditions. The resulting variational BIEs are as follows, for the Dirichlet elastic problem

$$
\text { Find } \boldsymbol{t} \in \mathcal{V}_{T} \quad \mathcal{B}_{t t}(\boldsymbol{t}, \tilde{\boldsymbol{t}})=\mathcal{L}_{t}(\tilde{\boldsymbol{t}}) \quad \forall \tilde{\boldsymbol{t}} \in \mathcal{V}_{T}
$$

with

$$
\begin{aligned}
\mathcal{B}_{t t}(\boldsymbol{u}, \tilde{\boldsymbol{t}})= & \int_{\partial \Omega} \int_{\partial \Omega} t_{k}(\boldsymbol{x}) \tilde{t}_{i}(\boldsymbol{y}) U_{i}^{k}(\boldsymbol{x}, \boldsymbol{y}) \mathrm{d} S_{y} \mathrm{~d} S_{x} \\
\mathcal{L}_{t}(\tilde{\boldsymbol{t}})= & \kappa \int_{\partial \Omega} \tilde{t}_{k}(\boldsymbol{x}) u_{k}^{D}(\boldsymbol{x}) \mathrm{d} S_{x} \\
& +\int_{\partial \Omega} \tilde{t}_{k}(\boldsymbol{y}) \int_{\partial \Omega}\left[u_{i}^{D}(\boldsymbol{x})-u_{i}^{D}(\boldsymbol{y})\right] n_{j}(\boldsymbol{x}) \Sigma_{i j}^{k}(\boldsymbol{y}, \boldsymbol{x}) \mathrm{d} S_{x} \mathrm{~d} S_{y}
\end{aligned}
$$

and the Neumann elastic problem:

$$
\text { Find } \boldsymbol{u} \in \mathcal{V}_{u} \quad \mathcal{B}_{u u}(\boldsymbol{u}, \tilde{\boldsymbol{u}})=\mathcal{L}_{u}(\tilde{\boldsymbol{u}}) \quad \forall \tilde{\boldsymbol{u}} \in \mathcal{V}_{u}^{0}
$$

with

$$
\begin{aligned}
\mathcal{B}_{u u}(\boldsymbol{u}, \tilde{\boldsymbol{u}})= & \int_{\partial \Omega}(R u)_{i q}(\boldsymbol{x}) \int_{\partial \Omega} B_{i q k s}(\boldsymbol{x}, \boldsymbol{y})(R \tilde{u})_{k s}(\boldsymbol{y}) \mathrm{d} S_{y} \mathrm{~d} S_{x} \\
\mathcal{L}_{u}(\tilde{\boldsymbol{u}})= & (\kappa-1) \int_{\partial \Omega} t_{k}^{D}(\boldsymbol{x}) \tilde{u}_{k}(\boldsymbol{x}) \mathrm{d} S_{x} \\
& +\int_{\partial \Omega} t_{k}^{D}(\boldsymbol{x}) \int_{\partial \Omega}\left[\tilde{u}_{i}(\boldsymbol{y})-\tilde{u}_{i}(\boldsymbol{x})\right] n_{j}(\boldsymbol{y}) \sum_{i j}^{k}(\boldsymbol{x}, \boldsymbol{y}) \mathrm{d} S_{y} \mathrm{~d} S_{x}
\end{aligned}
$$




\subsection{Plane elasticity}

The result (39) can be specialized to plane elasticity. In this case, formulae (41) to (45) remain valid, using the plane strain/stress counterpart of the 3D fundamental solution (the range of all indices is $\{1,2\}$ ).

Moreover, one readily sees that in eqn. (33) indices $i, j, k, l$ and $e, f, g, h$ should also range in $\{1,2\}$, which implies $p=q=r=s=3$. Thus:

$$
\begin{aligned}
C_{k l a b} \frac{\partial}{\partial z_{b}} \Sigma_{i j}^{a}(\boldsymbol{z}, \boldsymbol{x}) & =e_{3 e p} e_{3 f q} e_{3 g r} e_{3 h s} \frac{\partial}{\partial x_{e}} \frac{\partial}{\partial x_{f}} \frac{\partial}{\partial z_{g}} \frac{\partial}{\partial z_{h}} A_{3333}(\boldsymbol{z}, \boldsymbol{x}) \\
& =e_{e p} e_{f q} e_{g r} e_{h s} \frac{\partial}{\partial x_{e}} \frac{\partial}{\partial x_{f}} \frac{\partial}{\partial z_{g}} \frac{\partial}{\partial z_{h}} A(\boldsymbol{z}, \boldsymbol{x}) \quad(\boldsymbol{z} \neq \boldsymbol{x})
\end{aligned}
$$

for either plane strain or plane stress (with $e_{12}=-e_{21}-1, e_{11}=e_{22}=0$ ). Eqn. (40) then reduces to:

$$
\mathcal{B}_{u u}(\boldsymbol{u}, \tilde{\boldsymbol{u}})=\int_{S_{T}} \int_{S_{T}} \frac{d u_{i}}{d s}(\boldsymbol{x}) \frac{d \tilde{u}_{k}}{d s}(\boldsymbol{y}) e_{i e} e_{k g} \frac{\partial^{2}}{\partial x_{e} \partial y_{g}} A(\boldsymbol{x}, \boldsymbol{y}) \mathrm{d} s_{y} \mathrm{~d} s_{x}
$$

and a similar expression should be used in the relevant term of $\mathcal{L}_{u}$, eqn. (44).

For the two-dimensional isotropic plane strain Kelvin solution, one has (Bonnemay $[3]):$

$$
A(\boldsymbol{z}, \boldsymbol{x})=\frac{\mu}{4 \pi(1-\nu)} r^{2} \ln r \quad r=|\boldsymbol{z}-\boldsymbol{x}|
$$

\section{Indirect variational BIE formulation}

The direct formulation (39 to 45), being valid for both bounded or unbounded domains, can be used to derive an indirect formulation as well. Consider a bounded regular domain $\Omega$ together with its complement $\bar{\Omega}=\mathbb{R}^{3}-\Omega$ and denote by $\boldsymbol{u}^{I}, \boldsymbol{t}^{I}$ and $\boldsymbol{u}^{E}, \boldsymbol{t}^{E}$ respectively the solutions to the coupled interior and exterior elastostatic problems with equal prescribed displacements and opposite prescribed tractions:

$$
\boldsymbol{u}^{I}=\boldsymbol{u}^{E}=\boldsymbol{u}^{D} \quad \text { on } S_{u} \quad \boldsymbol{t}^{I}=-\boldsymbol{t}^{E}=\boldsymbol{t}^{D} \quad \text { on } S_{T}
$$

Let also $\boldsymbol{n}$ and $\overline{\boldsymbol{n}}$ denote the opposite normals to $\partial \Omega$, respectively outwards from $\Omega$ and $\bar{\Omega}$.

Then, the stationarity condition (16) is considered for the coupled problem. The test functions $\delta \boldsymbol{u}$ should be equal for both the interior and exterior problem, thus (14) and 
(15) should ultimately be taken with equal $\tilde{\boldsymbol{u}}, \tilde{\boldsymbol{t}}$ and the same unit normal, say $\boldsymbol{n}(\boldsymbol{y})$. This extends to eqns. (24), (25), (32), (38). On the other hand, the unit normal $\boldsymbol{n}(\boldsymbol{x})$ in the latter equations is the one that appears in $\boldsymbol{T}^{n}(\boldsymbol{\delta} \boldsymbol{u})$ in (5) and thus should change sign. Therefore, the variational formulation (39) becomes, for the coupled problem:

$$
\begin{aligned}
& \text { Find } \boldsymbol{u}^{E}, \boldsymbol{u}^{I} \in \mathcal{V}_{u}, \boldsymbol{t}^{E}, \boldsymbol{t}^{I} \in \mathcal{V}_{T} \\
& \qquad \begin{array}{r}
-\mathcal{B}_{u u}\left(\boldsymbol{u}^{E}, \tilde{\boldsymbol{u}}\right)+\mathcal{B}_{u u}\left(\boldsymbol{u}^{I}, \tilde{\boldsymbol{u}}\right)+\mathcal{B}_{t u}\left(\boldsymbol{t}^{E}, \tilde{\boldsymbol{u}}\right)+\mathcal{B}_{t u}\left(\boldsymbol{t}^{I}, \tilde{\boldsymbol{u}}\right)=-L_{u}(\tilde{\boldsymbol{u}}) \\
-\mathcal{B}_{u t}\left(\boldsymbol{u}^{E}, \tilde{\boldsymbol{t}}\right)+\mathcal{B}_{u t}\left(\boldsymbol{u}^{I}, \tilde{\boldsymbol{t}}\right)+\mathcal{B}_{t t}\left(\boldsymbol{t}^{E}, \tilde{\boldsymbol{t}}\right)+\mathcal{B}_{t t}\left(\boldsymbol{t}^{I}, \tilde{\boldsymbol{t}}\right)=L_{t}(\tilde{\boldsymbol{t}}) \\
\forall \tilde{\boldsymbol{u}} \in \mathcal{V}_{u}^{0}, \forall \tilde{\boldsymbol{t}} \in \mathcal{V}_{T}
\end{array}
\end{aligned}
$$

where $\mathcal{B}_{u u}$, etc. are given by (40), (41), (42), (43) with the normal $\boldsymbol{n}$ outwards from $\Omega$, while from (44), (45) many terms cancel out when appropriately combined, so that:

$$
L_{u}(\tilde{\boldsymbol{u}})=\int_{S_{T}} t_{i}^{D}(\boldsymbol{x}) \tilde{u}_{i}(\boldsymbol{x}) \mathrm{d} S_{x} \quad L_{t}(\tilde{\boldsymbol{t}})=\int_{S_{u}} u_{i}^{D}(\boldsymbol{x}) \tilde{t}_{i}(\boldsymbol{x}) \mathrm{d} S_{x}
$$

Let $\Delta \boldsymbol{u}=\boldsymbol{u}^{E}-\boldsymbol{u}^{I}$ and $\Delta \boldsymbol{t}=-\boldsymbol{t}^{E}-\boldsymbol{t}^{I}$ denote respectively the displacement and traction jumps accross $\partial \Omega$ (note that $\boldsymbol{t}^{I}$ and $\boldsymbol{t}^{E}$ are defined in terms of the opposite normals $\boldsymbol{n}$ and $\overline{\boldsymbol{n}}$, and also that $\Delta \boldsymbol{u}$ should be continuous over $\partial \Omega$ and satisfy (12)). Thus, due to the linearity of $\mathcal{B}_{u u}$, etc. w.r.t. each argument, the variational formulation for the coupled problem takes the following, clearly symmetric, form:

$$
\begin{aligned}
& \text { Find } \Delta \boldsymbol{u} \in \mathcal{V}_{u}^{0}, \Delta \boldsymbol{t} \in \mathcal{V}_{T} \\
& \qquad\left\{\begin{array}{r}
\mathcal{B}_{u u}(\Delta \boldsymbol{u}, \tilde{\boldsymbol{u}})+\mathcal{B}_{t u}(\Delta \boldsymbol{t}, \tilde{\boldsymbol{u}})=L_{u}(\tilde{\boldsymbol{u}}) \\
\mathcal{B}_{u t}(\Delta \boldsymbol{u}, \tilde{\boldsymbol{t}})+\mathcal{B}_{t t}(\Delta \boldsymbol{t}, \tilde{\boldsymbol{t}})=
\end{array} \quad \forall \tilde{\boldsymbol{u}} \in L_{t}(\tilde{\boldsymbol{t}})\right.
\end{aligned}
$$

One notices that the complexity of the right-hand sides is dramatically reduced between the direct formulation (39) and the indirect one (54), which means substantial savings in terms of computational time. However, it should be emphasized that, in order to recover the physical quantities $\boldsymbol{u}$ on $S_{u}$ and $\boldsymbol{t}$ on $S_{u}$, one still has to compute the appropriate integral representations, the limiting expression of:

$$
\begin{aligned}
u_{k}(\boldsymbol{z}) & =\int_{S_{T}} \tilde{u}_{i}(\boldsymbol{y}) n_{j}(\boldsymbol{y}) \Sigma_{i j}^{k}(\boldsymbol{z}, \boldsymbol{y}) \mathrm{d} S_{y}-\int_{S_{u}} \tilde{t}_{i}(y) U_{i}^{k}(\boldsymbol{z}, \boldsymbol{y}) \mathrm{d} S_{y} \\
\sigma_{i j}\left(\boldsymbol{z}^{\prime}\right) & =C_{k l a b} \int_{S_{T}} \tilde{u}_{k}(\boldsymbol{y}) n_{\ell}(\boldsymbol{y}) \frac{\partial}{\partial y_{b}} \Sigma_{i j}^{a}\left(\boldsymbol{y}, \boldsymbol{z}^{\prime}\right) \mathrm{d} S_{y}-\int_{S_{u}} \tilde{t}_{k}(y) \Sigma_{i j}^{k}\left(\boldsymbol{y}, \boldsymbol{z}^{\prime}\right) \mathrm{d} S_{y}
\end{aligned}
$$


for $\boldsymbol{z} \rightarrow \boldsymbol{x} \in S_{T}, \boldsymbol{z}^{\prime} \rightarrow \boldsymbol{x} \in S_{u}$, both leading to strongly singular integrals. Note that their computation thus relies on $\Delta \boldsymbol{u}, \Delta \boldsymbol{t}$ being both $C^{0, \alpha}$ at the point $\boldsymbol{x}$ of interest, while the assumption made on $\boldsymbol{t}$ for the direct formulation (39) is weaker. Also, there may be some cases for which the boundary elastic quantity themselves are only of secondary interest (e.g. in some fracture or optimization problems), giving a good reason for using the indirect formulation (54).

Another comment of interest is that, in the indirect case, the value $E$ at equilibrium (i.e. for $\Delta \boldsymbol{u}, \Delta \boldsymbol{t}$ solution to (54)) of the elastic potential energy is given (e.g. from (3)) by:

$$
E=\frac{1}{2}\left[L_{t}(\Delta \boldsymbol{t})-L_{u}(\Delta \boldsymbol{u})\right]
$$

Thus the

Formulation (54) applied to the special case of the Dirichlet elastic problem gives:

Find $\Delta \boldsymbol{t} \in \mathcal{V}_{T}, \forall \tilde{\boldsymbol{t}} \in \mathcal{V}_{T}$

$$
\int_{\partial \Omega} \int_{\partial \Omega} \psi_{k}(\boldsymbol{x}) \tilde{t}_{i}(\boldsymbol{y}) U_{i}^{k}(\boldsymbol{x}, \boldsymbol{y}) \mathrm{d} S_{y} \mathrm{~d} S_{x}=\int_{\partial \Omega} \tilde{t}_{k}(\boldsymbol{x}) u_{k}^{D}(\boldsymbol{x}) \mathrm{d} S_{x}
$$

while one has for the Neumann elastic problem:

Find $\Delta \boldsymbol{u} \in \mathcal{V}_{u}^{0}, \forall \tilde{\boldsymbol{t}} \in \mathcal{V}_{u}^{0}$

$$
\int_{\partial \Omega}(R \phi)_{i q}(\boldsymbol{x}) \int_{\partial \Omega} B_{i q k s}(\boldsymbol{x}, \boldsymbol{y})(R \tilde{u})_{k s}(\boldsymbol{y}) \mathrm{d} S_{y} \mathrm{~d} S_{x}=\int_{\partial \Omega} \tilde{u}_{k}(\boldsymbol{x}) t_{k}^{D}(\boldsymbol{x}) \mathrm{d} S_{x}
$$

The two above formulations may be found e.g. in Nedelec [15].

\section{Numerical implementation}

The symmetry properties (46) allow a reduction of computational effort for the linear system solution step due to the symmetric character of the governing matrix; this has been extensively discussed elsewhere. We wish to focus the discussion on the treatment of the singular surface integrals in (40) to (45), i.e. repeated integrals over the same element $E$, for the simplest case of isoparametric conformal BEM interpolation. The surface $\partial \Omega$ is divided into boundary elements, which are mapped on a reference element $E_{0}$, (usually either the square $\boldsymbol{\xi}=\left(\xi_{1}, \xi_{2}\right) \in[-1,1]^{2}$ or the triangle $\left.0 \leq \xi_{1}+\xi_{2} \leq 1\right)$. Let the discussion be restricted to the consideration of a single element $E$. The location of a point $\boldsymbol{y}$ on $E$ is 
expressed in terms of $N$ shape functions $N^{K}$ and $N$ geometrical nodes $\boldsymbol{y}^{K}(K=1 \ldots n)$ located on the boundary of $E$ :

$$
\boldsymbol{y}=\sum_{K=1}^{N} N^{K}(\boldsymbol{\xi}) \boldsymbol{y}^{K} \quad\left(\boldsymbol{\xi} \in E_{0}\right)
$$

Then, for $(\alpha, \beta)=1,2$, the natural basis $\left(\mathbf{a}_{\alpha}\right)$, metric tensor $\left(g_{\alpha \beta}\right)$ and unit normal $\boldsymbol{n}$ on $E$ are given by:

$$
\begin{array}{r}
\boldsymbol{a}_{\alpha}(\boldsymbol{\xi})=\sum_{K=1}^{N} N_{, \alpha}^{K}(\boldsymbol{\xi}) \boldsymbol{y}^{K} \quad g_{\alpha \beta}(\boldsymbol{\xi})=\boldsymbol{a}_{\alpha}(\boldsymbol{\xi}) \cdot \boldsymbol{a}_{\beta}(\boldsymbol{\xi}) \\
J(\boldsymbol{\xi}) \boldsymbol{n}(\boldsymbol{\xi})=\boldsymbol{a}_{\mathbf{1}} \wedge \boldsymbol{a}_{\mathbf{2}} \quad J^{2}(\boldsymbol{\xi})=\left(g_{11} g_{22}-g_{12}^{2}\right)(\boldsymbol{\xi})
\end{array}
$$

The shape functions $N^{K}$ are also used to interpolate $\boldsymbol{u}, \boldsymbol{t}, \tilde{\boldsymbol{u}}, \tilde{\boldsymbol{t}}$. Moreover, it can be shown that:

$$
\forall \boldsymbol{\xi} \in E_{0} \quad e_{a b c} n_{b}(\boldsymbol{y}) \frac{\partial f}{\partial y_{c}}(\boldsymbol{y}) J(\boldsymbol{\xi}) \boldsymbol{e}_{a}=\frac{\partial f}{\partial \xi_{1}} \boldsymbol{a}_{2}-\frac{\partial f}{\partial \xi_{2}} \boldsymbol{a}_{1} \quad(\boldsymbol{y}=\boldsymbol{y}(\boldsymbol{\xi}))
$$

Let $\boldsymbol{\eta}=\left(\eta_{1}, \eta_{2}\right)$ denote the antecedent of $\boldsymbol{x}$ on $E_{0}$. Following a common practice in BEM (see e.g. Rizzo et al. [20]), set $\xi_{1}=\eta_{1}+\rho \cos \alpha, \xi_{2}=\eta_{2}+\rho \sin \alpha$. Then:

$$
\mathrm{d} S_{y}=J(\boldsymbol{\xi}) d \xi_{1} d \xi_{2}=J(\boldsymbol{\xi}) \rho d \rho d \alpha
$$

Define $\hat{N}^{q}(\rho, \alpha ; \boldsymbol{\eta}), \hat{r}(\rho, \alpha ; \boldsymbol{\eta}), \hat{U}_{i}^{k}(\rho, \alpha ; \boldsymbol{\eta}), \hat{\Sigma}_{i s}^{k}(\rho, \alpha ; \boldsymbol{\eta}), \hat{B}_{i q k s}(\rho, \alpha ; \boldsymbol{\eta})$ as follows:

$$
\begin{gathered}
N^{K}(\boldsymbol{\xi})-N^{K}(\boldsymbol{\eta})=\rho \hat{N}^{K}(\rho, \alpha ; \boldsymbol{\eta}) \\
r(\boldsymbol{x}, \boldsymbol{y})=\left\|\sum_{K=1}^{K} N^{K}(\boldsymbol{\xi}) \boldsymbol{y}^{K}\right\|=\rho \hat{r}(\rho, \alpha ; \boldsymbol{\eta}) \\
U_{i}^{k}(\boldsymbol{x}, \boldsymbol{y})=\frac{1}{\rho} \hat{U}_{i}^{k}(\rho, \alpha ; \boldsymbol{\eta}) \\
\sum_{i s}^{k}(\boldsymbol{x}, \boldsymbol{y})=\frac{1}{\rho^{2}} \hat{\Sigma}_{i s}^{k}(\rho, \alpha ; \boldsymbol{\eta}) \\
B_{i q k s}(\boldsymbol{x}, \boldsymbol{y})=\frac{1}{\rho} \hat{B}_{i q k s}(\rho, \alpha ; \boldsymbol{\eta})
\end{gathered}
$$

where $\hat{r}(\rho, \alpha ; \boldsymbol{\eta}) \neq 0$ for sufficiently small $\rho$ and $\hat{\boldsymbol{U}}(\rho, \alpha ; \boldsymbol{\eta}), \hat{\boldsymbol{\Sigma}}(\rho, \alpha ; \boldsymbol{\eta}), \hat{B}_{i q k s}(\rho, \alpha ; \boldsymbol{\eta})$ are thus regular at $\rho=0$. Hence typical (inner) singular surface integrals in (40) to (45) 
become:

$$
\begin{aligned}
& \int_{E} n_{s}(\boldsymbol{y}) \Sigma_{i s}^{k}(\boldsymbol{x}, \boldsymbol{y})\left(u_{i}(\boldsymbol{y})-u_{i}(\boldsymbol{x})\right) \mathrm{d} S_{y} \\
& =\sum_{K=1}^{N} u_{i}^{K} \int_{E} n_{s}(\boldsymbol{y}) \hat{\Sigma}_{i s}^{k}(\rho, \alpha ; \boldsymbol{\eta}) \hat{M}^{K}(\rho, \alpha ; \boldsymbol{\eta}) J(\boldsymbol{\xi}) d \rho d \alpha \\
& \int_{E} B_{i q k s}(\boldsymbol{x}, \boldsymbol{y}) e_{l h s} n_{l}(\boldsymbol{y}) u_{k, h}(\boldsymbol{y}) \mathrm{d} S_{y} \\
& =\sum_{K=1}^{N} u_{i}^{K} \int_{E} \hat{B}_{i q k s}(\rho, \alpha ; \boldsymbol{\eta})\left[\left(\boldsymbol{a}_{\boldsymbol{\beta}} \cdot \boldsymbol{e}_{\boldsymbol{s}}\right) M_{, \alpha}^{K}-\left(\boldsymbol{a}_{\boldsymbol{\alpha}} \cdot \boldsymbol{e}_{\boldsymbol{s}}\right) M_{, \beta}^{K}\right] d \rho d \alpha \\
& \int_{E} U_{i}^{k}(\boldsymbol{x}, \boldsymbol{y}) t_{i}(\boldsymbol{y}) \mathrm{d} S_{y} \\
& =\sum_{K=1}^{N} t_{i}^{K} \int_{E} \hat{U}_{i}^{k}(\rho, \alpha ; \boldsymbol{\eta}) M^{K}(\boldsymbol{\xi}) J(\boldsymbol{\xi}) d \rho d \alpha
\end{aligned}
$$

Equations (60), (61), (62) take full advantage of the regularization. A further coordinate change $(\rho, \alpha) \rightarrow\left(v_{1}, v_{2}\right)$, in order to convert them to integrals over the square $[-1,1]^{2}$ [4], allows their numerical evaluation to be performed with standard product Gaussian quadrature formulas.

The double surface integrals over $E \times E$ are computable by using standard Gaussian quadrature (points $\boldsymbol{\eta}_{i}$, weights $w_{i}$ ) for the outer one:

$$
I_{e, e}^{\text {sing }}=\int_{E_{0}} \int_{E_{0}} f(\boldsymbol{\eta}, \boldsymbol{\xi}) \mathrm{d} \eta \mathrm{d} \xi \approx \sum_{i=1}^{n} w_{i} \int_{E_{0}} f\left(\boldsymbol{\eta}_{i}, \boldsymbol{\xi}\right) \mathrm{d} \xi
$$

The remaining, weakly singular, integral is evaluated using formulas (60), (61), (62).

\section{Concluding comments.}

In this paper, symmetric variational regularized BIE formulations are established, for the mixed elastostatic boundary-value problem. A direct version (in terms of unknown boundary displacements and tractions) is first presented. Then an indirect version (in terms of unknown fictitious densities) is in turn established. Both are expressed using at most weakly singular integrals followed by regular integrals. These formulations provide a basis for the numerical solution of general 3D situations. Conventional boundary element interpolations of any degree for the geometry as well as the unknowns can be implemented in a straightforward manner, using conventional techniques for numerical 
integration together with a now well-established singularity cancellation method at the shape function level.

Moreover, the variational BIE formulations are shown to express the stationarity of an augmented potential energy functional, thus being truly a variational BIE formulation.

The present approach can be developed to other situations, such as potential problems or elastodynamic problems, as well. Moreover, similar variational BIE formulations are already known in the literature, notably for Dirichlet or Neumann problems in various contexts, including crack problems (see references quoted herein).

\section{References}

[1] BeCACHE E. - Résolution par une méthode d'équations intégrales d'un problème de diffraction d'ondes élastiques transitoires par une fissure. Thesis, Université Paris 6, France, 1991.

[2] Bendali A. - Problème aux limites extérieur et intérieur pour le système de Maxwell en régime harmonique. Internal report no. 59, Centre de Mathématiques Appliquées, Ecole Polytechnique, Palaiseau, France, 1980

[3] P. Bonnemay - Equations intégrales pour l'élasticité plane. Thesis, Université Paris VI, France, 1979.

[4] Bonnet M. - Méthode des équations intégrales régularisées en élastodynamique tridimensionnelle. Thesis, Ecole Nationale des Ponts et Chaussées, Paris, France, 1986.

[5] Bonnet M. - Regularized Boundary Integral Equations for Three-dimensional Bounded or Unbounded Elastic Bodies Containing Curved Cracks of Arbitrary Shape Under Dynamic Loading. In Boundary Element Techniques: Applications in Engineering (C.A. Brebbia, N.G. Zamani, eds.), Computational Mechanics Publications (Southampton), 1989.

[6] Bonnet M. And Bui H.D. - Regularization of the Displacement and Traction BIE for 3D Elastodynamics using indirect methods. In: Advances in Boundary Element 
Techniques, J.H. Kane, G. Maier, N. Tosaka and S.N. Atluri, eds., Springer-Verlag, 1993.

[7] Bui H.D. - On the variational boundary integral equations in elastodynamics with the use of conjugate functions. J. Elast. 28, 247-256, 1992.

[8] Cortey-Dumont PH. - Simulation numérique de problèmes de diffraction d'ondes par une fissure. Thesis, Université Paris VI, France, 1985.

[9] Guighiani M. , Krishnasamy G. , Rudolphi T.J., Rizzo F.J. - A general algorithm for the numerical solution of hypersingular boundary integral equations. ASME J. Appl. Mech. , -, 1992.

[10] Hamdi M.A. - Formulation variationnelle par équations intégrales pour le calcul de champs acoustiques linéaires proches et lointains. Thesis, Université Technologique de Compiègne, France, 1982.

[11] Kane J.H., Balakrishna C. - Symmetric Galerkin boundary formulations employing curved elements. Int. J. Num. Meth. in Eng,. 36, 2157-2187, 1993.

[12] Balakrishna C., Gray L.J., Kane J.H. - Efficient analytical integration of symmetric Galerkin boundary integrals over curved elements: elasticity formulation. Comp. Meth. in Appl. Mech. Engng., to appear, 1994.

[13] Krishnasamy G., Rizzo F.J., Rudolphi T.J. - Hypersingular boundary integral equations: their occurrence, interpretation, regularization and computation. In P.K. Banerjee and S. Kobayashi (eds.), Developments in Boundary Element Methods - vol. 7: Advanced Dynamic Analysis, chap. 7, Elsevier, 1992.

[14] K - upradze V.D. (Editor) - Three-dimensional problems of the mathematical theory of elasticity and thermoelasticity. North Holland, 1979.

[15] NedeleC J.C. - Integral equations with non integrable kernels. Integral equations and operator theory, vol. 5, pp 562-572, 1982.

[16] Nishimura N, Kobayashi S. - A regularized boundary integral equation method for elastodynamic crack problems. Comp. Mech. 4, 319-328, 1989. 
[17] Nishimura N, Kobayashi S. - A boundary integral equation method for an inverse problem related to crack detection. Int. J. Num. Meth. in Eng,. 32, 1371-1387, 1991.

[18] Parreira P., Guiggiani M. - On the implementation of the Galerkin approach in the boundary element method. Computers and Structures 33, 269-279, 1989.

[19] Polizzotto C. - An energy approach to the boundary element method. Part I: elastic solids. Comp. Meth. in Appl. Mech. Engng. 69, 167-184, 1988.

[20] Rizzo F.J., Shippy D.J., Rezayat M. - A boundary integral equation method for radiation and scattering of elastic waves in three-dimensions. Int. J. Num. Meth. in Eng,. 21, 115-129, 1985.

[21] Sirtori S., Maier G., Novati G., Miccoli S. - A Galerkin symmetric boundary element method in elasticity: formulation and implementation. Int. J. Num. Meth. in Eng,. 35, 255-282, 1992.

[22] Wendland W.L. - Mathematical properties and asymptotic error estimates for elliptic boundary element methods. In T.A. Cruse (ed.), Advanced boundary element methods, pp. 475-489, Springer-Verlag, 1988. 